\title{
Investigation of cost, power, and spectral efficiency in fixed- and flexi-grid networks
}

\author{
Sridhar Iyer ${ }^{1 *}$, Shree Prakash Singh ${ }^{2}$ \\ 1. Department of ECE, Jain College of Engineering, T S. Nagar-Machhe, Belagavi, Karnataka 590014, India \\ 2. Division of ECE, Netaji Subhas Institute of Technology, Sector-3, Dwarka, New Delhi 110078, India \\ * Corresponding author, Email: sridhariyer1983@gmail.com
}

\begin{abstract}
With the steady growth of traffic volume in core networks, it is predicted that future optical network communication will be constrained mainly by cost and power consumption. Thus, for Internet sustainability, it will be necessary to ensure cost and power efficiency in optical networks. The aims of this study are (i) to identify the main sources of cost and power consumption in fixed-grid (SLR and MLR) and flexi-grid (OFDM) optical networks, and (ii) to compare techniques for improving cost and power efficiency in SLR/MLR- and OFDM-based networks. To this end, we conducted a comparative analysis of cost and power efficiency for the OFDM- and MLR/SLR-based networks, and considering realistic networks, evaluated the cost and power consumed by different components in the optical layer. Our results show that (i) OFDM-based networks outperform those based on MLR/SLR in terms of both cost and power-efficiency, (ii) the extra equipment cost incurred due to under-utilization of spectrum is reduced by switching to a flexi-grid network, (iii) lower power consumption per bit is obtained when the networking solution ensures a finer bit-rate granularity, and (iv) there exists a power and spectrum minimization trade-off that is network characteristic dependent.
\end{abstract}

Keywords: mixed line rate, single line rate, OFDM, fixed- and flexi-grid, cost-efficiency, power-efficiency, spectral-efficiency

\section{Introduction}

To satisfy the demands of heterogeneous services having different applications and varied bandwidth requirements, with the adoption of a MLR (Mixed Line Rate) strategy, the legacy $10 \mathrm{Gbit} / \mathrm{s}$ transport optical networks have been upgraded to 40 and/or $100 \mathrm{Gbit} / \mathrm{s}$ networks ${ }^{[1]}$. Within the same fiber, the co-existence of 10/40/100 Gbit/s on varied wavelengths can be provided, and the overall transmission cost can be decreased owing to the volume discount of high bit-rate transponders ${ }^{[2]}$. In fixed-grid (i.e., both SLR (Single Line Rate) and MLR) networks, a particular transceiver type is assumed and only a single demand serving method exists, which fixes the bit-rate, TR (Transmission Reach), and spectrum utilized. However, such networks are based on the fixed grid as defined by the ITU-T (International Telecommunications Union Telecommunication Standardization Sector), and hence are required to admit all channels within a fixed $50 \mathrm{GHz}$ channel spacing ${ }^{[2]}$. This may not be adequate for high-speed channels, and may under-utilize spectrum for low bit rate requests. Hence, in pursuing technologies for future optical networks, it is essential that line rates greater than $100 \mathrm{Gbit} / \mathrm{s}$ be supported, which can 
be accomplished by using flexi-grid systems. Networks based on the flexi-grid system can (i) adjust bandwidth utilization according to demand, (ii) provide long TR and high SE (Spectral-Efficiency), (iii) add flexibility in traffic and network engineering, and (iv) restore failed optical paths ${ }^{[3,4]}$. Such networks make use of flexible transceivers (or BVTs (Bandwidth Variable Transponders)) that support several demand serving options by making decisions on the modulation format, bit rate, and/or spectrum, and provide adequate distance reaching performance. Hence, a BVT with cost and tuned to Gbit/s transmission rate, and using spectrum slots of bandwidth and guard band, leads to amount of power consumed in order to transmit with a satisfactory QoT (Quality of Transmission) for $\mathrm{km}$ of distance. However, the deployment of such systems over the existing infrastructure incurs substantial CapEx (Capital Expenditure) owing to the requirement of software-driven and bandwidth-flexible (variable) components such as BVTs and OXCs (Optical Cross-Connects) ${ }^{[5]}$.

Recent studies have identified OFDM (Orthogonal Frequency-Division Multiplexing) as the enabling technology for flexi-grid system networks ${ }^{[6-11]}$. In OFDM, several orthogonal carriers (each individual carrier is referred to as a subcarrier $\left.{ }^{[4]}\right)$ are modulated, and the composite signal is then carried over an individual wavelength in a fiber. Further, many such wavelengths are multiplexed within the fiber. Compared to a single carrier network (i.e., SLR or MLR), which uses a fixed $50 \mathrm{GHz}$ grid, an OFDMbased flexi-grid network has the following features, as shown in Fig. 1: (i) the ITU-T-defined standardized granularity of $12.5 \mathrm{GHz}^{[12]}$ is followed and, on the basis of requirements, wider channels are created by combining the spectrum units (also called slots); (ii) multiple subcarriers are used, which ensures that the wavelength capacity can be zoned into finer granularities, thus providing increased flexibility in capacity allocation for heterogeneous demands. An OFDM signal can be generated electronically, referred to as E-OFDM (Electrical-OFDM), or optically, referred to as O-OFDM (Optical-OFDM $)^{[13]}$. Hence, over- all, in OFDM-based networks, owing to the flexible (or elastic) link types, effective demand bundling is provisioned. Further, less over-provisioning of network resources is required, resulting in both cost and power (or energy) efficiency (CE or PE).

In general, the design of optical networks targets CapEx minimization by using RWA (Routing and Wavelength Assignment) or RMLSA (Routing Modulation Level and Spectrum Allocation) algorithms, which are used for connection establishment in fixedand flexi-grid networks, respectively ${ }^{[2,6]}$. The RWA algorithms ensure that the wavelength continuity and single wavelength assignment constraints are met in a fixed-grid network, whereas in flexi-grid networks, in addition to the aforementioned constraints, the RMLSA algorithms ensure that the maximum subcarrier constraint is also met. In this constraint, the largest transparent TR limits the highest channel capacity, and hence, on the same channel, the maximum number of subcarriers. For both types of algorithms, RP (Regenerator Placement) is a subproblem, and, unlike fixed-grid networks, RP in flexigrid networks is more complicated because the selection of BVT configurations is also required. Network operators currently and in the future will attempt migration to next-generation networks by looking for the "best" solution that takes into account cost and performance. In recent years, due to considerations of the environment, economy, and future growth, power consumption has become a key factor because it affects the network OpEx (Operational Expenditure $)^{[14,15]}$. With the steady growth of Internet traffic and deployment and upgrading of network infrastructure, it is apparent that power consumption will place a major constraint on the Internet growth. Further, CapEx and power consumption are correlated; however, they are not always linearly related $^{[16]}$; hence, the power-efficient design of optical networks will require more investigation.

In the majority of previous studies, results relating to cost (CapEx) and power for various networking options have only been obtained individually. In this work, we perform a $\mathrm{CE}$ and PE comparison of backbone optical networks with SLR or MLR (fixed-grid) 


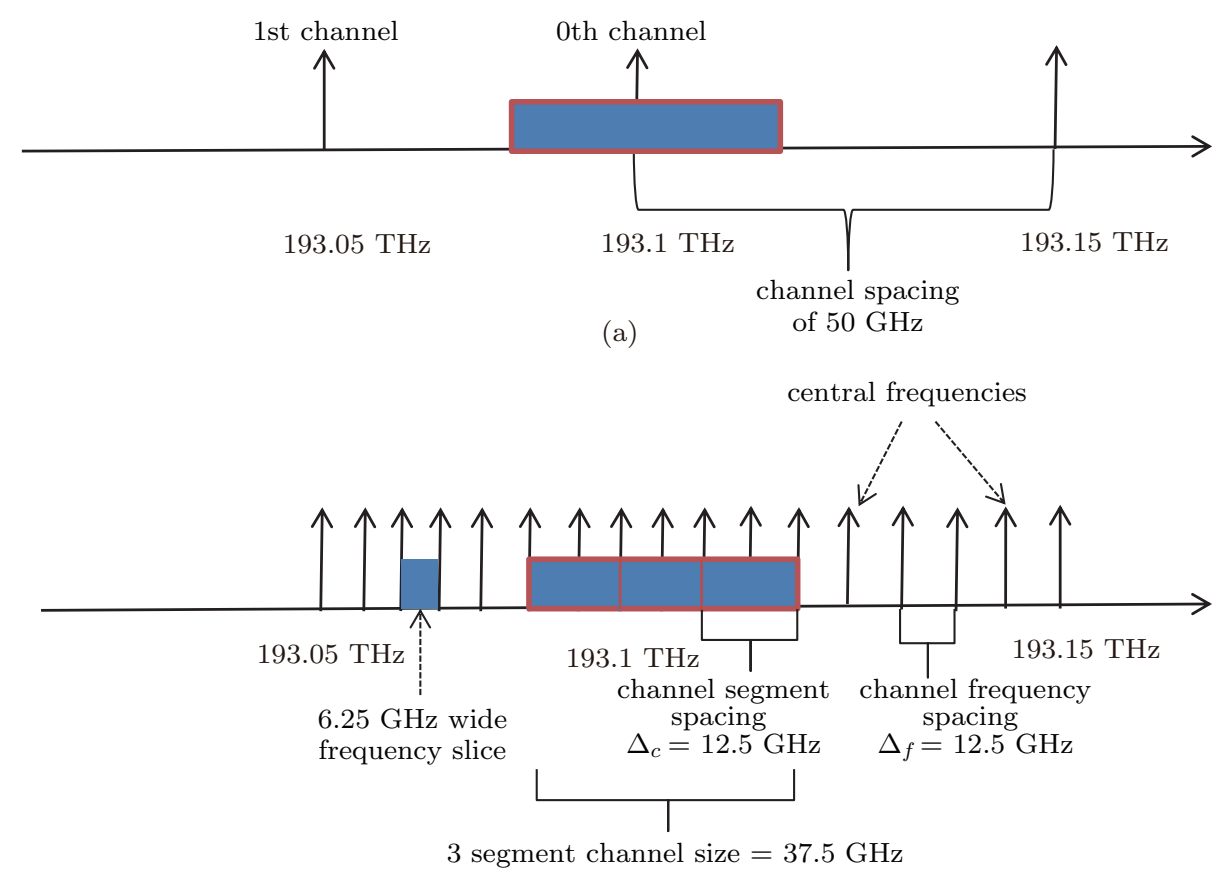

(b)

Figure 1 Fixed-grid strategy with optical channel assigned two fixed spectrum widths: (a) $50 \mathrm{GHz}$; (b) $37.5 \mathrm{GHz}$

and O-OFDM (flexi-grid) as their enabling technologies. Our main objectives are to evaluate these emergent technologies in the transport networks from the perspectives of cost and power, and to provide a broad view of the potential of each solution. For this purpose, we utilize power consumption and cost parameters of realistic components in order to calculate cost and power consumption with high accuracy for actual cases. Our current work distinguishes itself from previous studies as follows. We use an innovative PA-RSA (Power Aware Routing and Spectrum Allocation) algorithm for the flexi-grid networks that considers the various transponder transmission parameters and harvests their tunability to improve the PE of the network. Moreover, to obtain the desired results, we simulate realistic network topologies and their corresponding traffic matrices. Hence, we argue that the conclusions drawn from our study are more accurate and applicable than conclusions obtained from the majority of previous studies.

The rest of this paper is structured as follows. In section 2 , studies related to this work are presented. Section 3 presents the cost and power models used in the fixed- and flexi-grid networks. In section 4, we detail the algorithms used in the study. Section 5 presents and discusses the various simulation results obtained. Finally, the conclusions of the study are presented in section 6 .

\section{Related works}

A detailed survey of various design studies for fixedgrid networks, both SLR and MLR, is presented in Ref. [2]. Further, in our previous work ${ }^{[17]}$, we investigated the SE and PE of SLR and MLR networks. Our results indicated that (i) for large capacity networks, the combination of a $100 \mathrm{Gbit} / \mathrm{s}$ transponder and $40 \mathrm{Gbit} / \mathrm{s}$ regenerator has the largest PE; (ii) for different SLR and MLR cases and longer distances, a merging point in efficiency occurs; and (iii) for the MLR case, improvement in the SE and PE can be achieved by dedicating shorter links and assigning high bandwidth to $100 \mathrm{Gbit} / \mathrm{s}$ wavelengths, or by using longer links and assigning high bandwidth to 40 Gbit/s wavelengths. The results also indicated that more power is consumed when the sole focus is on SE.

Recently, studies have also reported on various is- 
sues concerning flexi-grid OFDM-based networks. In Ref. [6], the authors compared fixed-grid and OFDM network architectures by proposing a physical layer impairment-aware routing and spectral allocation algorithm. The results showed that the advantages of the flexible-OFDM solutions are dependent on constraints imposed by the routing and characteristics of the traffic. The authors in Ref. [7] have compared the $\mathrm{CE}$ for optical networks that are based on mixed bit-rates (10/40/100 Gbit/s) and flexible solutions (25/50/100 Gbit/s) for translucent networks, considering the cost of only the opto-electronic interfaces. Further, the study based on OFDM investigated the PE for shorter TR optical interconnects, which are only suitable for connections at the intradata-center levels. In Ref. [8], the authors formulated a model based on mixed integer linear programming that minimizes the IP (Internet Protocol) consumed on a variable OFDM network. However, the model considered only the power consumption of the router ports, EDFAs (Erbium-Doped Fiber Amplifiers), and tunable OFDM transponders. In Ref. [9], for static traffic cases based on MLR and SLR networks, the authors proposed heuristic algorithms for evaluating the power consumption of flexible OFDM networks. It was shown that the algorithms service requests in a successive order by selecting the best power-efficient choice. The authors in Ref. [10] studied the PE of elastic networks with constraints on survivability, and compared the scheme of dedicated protection with that of shared protection. In Ref. [11], to minimize total consumed power, the authors examined the use of traffic grooming, format modulation (which is adaptive), and spectrum allocation (which is flexible). The authors formulated the minimization problem using integer linear programming followed by the formulation of a heuristic algorithm.

The studies mentioned above have reported results for the various networking solutions only individually for cost (CapEx) and power. Our main contributions that distinguish the current work from the earlier studies are (i) the development of detailed power and CapEx models that assess the power and cost of different components deployed in the fixedand the flexi-grid networks, and (ii) use of algorithms that take into account cost and power minimization jointly, and consider a complete set of available options before employing exact models to evaluate the cost and power consumed.

\section{Network models}

The power and CapEx model used in this study is shown in Fig. 2, which includes the equipment used for the fixed- and flexi-grid cases. It must be noted that even though more cost and power savings are obtained by optimizing both the optical and electronic layers, currently, the telecom operators optimize each layer separately. Hence, in this study we focus on investigation of $\mathrm{CE}$ and $\mathrm{PE}$ for the optical layer only, and it is assumed that the functionality of additional optical grooming is not available, i.e., we neglect any electronic traffic collection at the network edges.

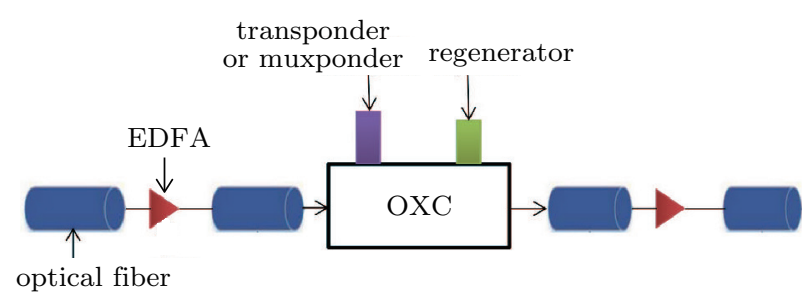

Figure 2 Power and cost model used in the study

\subsection{Network architecture}

In the optical layer, the nodes or switches are interconnected by bidirectional link pairs, and every link has one fiber supporting transmission over many wavelengths. The transmission begins and ends at associated nodes with specified ports and can optically bypass intermediate nodes. For traffic that originates/terminates at a node, a transponder, consisting of a receiver-transmitter pair, receives/transmits data via optical wavelength, and adapts the signal into a suitable form for transmission over the network.

The fiber link model used in the study is shown in Fig. 3, with the link separated into spans consisting 
of segments of SSMF (Standard Single Mode Fiber). Owing to attenuation, an amplifier is deployed at the end of each span, thus providing compensation for losses from the previous span. Further, we assume that the optical layer uses coherent transmission; hence, a dispersion compensation fiber is not utilized. The nodes consist of the following components: (i) WSSs (Wavelength Selective Switches) ${ }^{[4,5]}$ that implement the route and select the architecture ${ }^{[18]}$, (ii) EDFAs, and (iii) passive combiners and splitters. These components collectively form the NIs (Network Interfaces) used for connecting nodes with both the links and the add/drop terminals. Such types of nodes, which are remotely configurable, are also known as OXCs whose add/drop ports can be colorless (C)/directionless (D)/contentionless (C). In this study, we use an OXC architecture with the $\mathrm{C} / \mathrm{D} / \mathrm{C}$ feature, since such an OXC demonstrates the best lightpath blocking probability performance ${ }^{[19]}$. The cost and power consumption of OXC used in our study is evaluated using the following equation, which is adopted from the study in Ref. [20]:

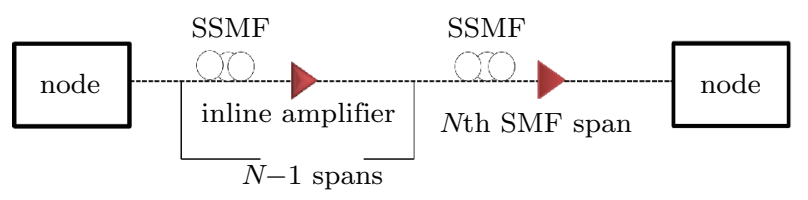

Figure 3 Model of the link used in the study

$$
\begin{aligned}
\text { Powerorcost }= & N \times(2 \times W S S+A m p)+ \\
& 2 \times W S S \times N \times A D(\%) / 20(\%)+ \\
& 2 \times W S S,
\end{aligned}
$$

where $N$ is degree of the OXC, Amp the power or cost consumption of two stage EDFAs, WSS the power or cost consumption of the WSS used (we assume the use of $1 \times 20 / 20 \times 1$ type $W S S)$, and $A D(\%)$ the percentage of add-drop of the OXC which is evaluated based on traffic which crosses OXC having a granularity of $20 \%$. Hence, optical layer consists of the following components: (i) transponders (fixed or variable for fixed-grid or flexi-grid network, respectively), (ii) regenerators, (iii) OXCs, and (iv) in-line EDFAs.

\subsection{Cost and power models}

The cost model in our study considers both power and cost (CapEx) consumption. Because the current state of the art in transponder technology is the coherent $100 \mathrm{Gbit} / \mathrm{s}^{[21]}$, for CapEx we use the $100 \mathrm{Gbit} / \mathrm{s}$ coherent transponder cost as the reference UC (Unit Cost), i.e., we price all the devices with reference to the UC. For the power consumption of the optical network components, we referred to various research studies, datasheets and project deliverables ${ }^{[21-37]}$, and we included the cost that arises owing to this power consumption.

1. Fixed-grid networks. For the fixed-grid (MLR/SLR) networks, the following components lead to power and cost consumption: (i) transponders, (ii) regenerators, (iii) OXCs that consist of WSSs, and (iv) EDFAs. The major components included within a transponder and the configuration of a regenerator are as detailed in Ref. [17]; further, a corresponding regenerator is available for every transponder. It is assumed that only transponders with line rates of 40/100/400 Gbit/s are available, i.e., we exclude the use of both $10 \mathrm{Gbit} / \mathrm{s}$ transponders, which are based on incoherent reception and require strategies for dispersion compensation, and $1 \mathrm{Tbit} / \mathrm{s}$ transponders, which will not appear in the immediate future ${ }^{[21]}$. For the components that form the OXCs, the power and cost contribution is considered through Eq. (1). We assume that the amplification span is $80 \mathrm{~km}$, and hence, after every span, we consider the deployment of EDFAs. With this model, and the methodology detailed in our previous study ${ }^{[17]}$, we evaluate the power and cost consumptions of the different components, and present the results in Tab. 1. It must be noted that the TR values presented in Tab. 1 are evaluated considering modulation formats as in Ref. [38], which account for the interference effects between various modulation formats and/or the bit-rates used in an MLR network.

2. Flexi-grid networks. The BVTs serve the traffic in the flexi-grid networks. The BVT model in our study is that of a muxponder ${ }^{[5]}$ with a maximum rate 
Table 1 Power and cost consumption values of various fixed-grid network components

\begin{tabular}{cccccc}
\hline architecture & bit rate & equipment & TR (km) & power (W) & cost (UC) \\
\hline- & $40 \mathrm{Gbit} / \mathrm{s}$ & $\mathrm{T} / 3 \mathrm{RR}$ & $2300 / 2300$ & $800 / 600$ & $0.68 / 0.91$ \\
\hline- & $100 \mathrm{Gbit} / \mathrm{s}$ & $\mathrm{T} / 3 \mathrm{RR}$ & $2100 / 2100$ & $1200 / 1400$ & $1 / 1.8$ \\
\hline- & $400 \mathrm{Gbit} / \mathrm{s}$ & $\mathrm{T} / 3 / \mathrm{RR}$ & $790 / 790$ & $1650 / 2100$ & $1.52 / 2.56$ \\
WSS & - & $\mathrm{O}$ & - & 120 & 0.68 \\
EDFA & - & $\mathrm{X}$ & - & 125 & 0.1 \\
S/C & - & $\mathrm{C}$ & - & 0 & 0 \\
\hline
\end{tabular}

T/3RR corresponds to Transponder/3R Regenerator and S/C corresponds to Splitter/Combiner.

of $400 \mathrm{Gbit} / \mathrm{s}$. Its main advantage is that it relies on a variety of TRs, thus providing the possibility of skipping intermediate regenerators whenever necessary. Following the study presented in Ref. [39], the BVT model consists of a variable (dynamic) part, that depends on the number of subcarriers allocated for every lightpath, and a fixed (static) part, that accounts for the cost/power of the transponder. The fixed part of the BVT is the major power consumer. The variable part of the BVT can change according to the flexible bandwidth, as various numbers of subcarriers modulated at appropriate levels (which can be DP-QPSK or DP- $n$-QAM $(n=8,16))$, are turned on. We extend the fixed bit-rate transponder model from our previous study ${ }^{[17]}$ and assume the following components in our BVT model: (i) client side, with client cards and framer/deframer (at each instant a client card being active or not depends on the transponder transmission rate); (ii) $\mathrm{E} / \mathrm{O}$ modulation, with drivers, laser, and local oscillator; and (iii) $\mathrm{O} / \mathrm{E}$ receiver, with photodiode, ADC, and DSP. We assume that the BVT transponder architecture consists of two lasers that transmit up to a maximum of $400 \mathrm{Gbit} / \mathrm{s}$; hence, the power consumption always arises from a specific number of lasers being active. Further, with the exception of the framer/deframer and DSP, which have a baud rate dependency, all other components have fixed power consumption. To evaluate the power consumed by the BVT transponder, we use the study detailed in Ref. [37] and form the following equation:

$$
P_{\mathrm{BVT}}=n \times\left(P_{\text {static }}+R \times P_{\text {dynamic }}\right) \times P_{\text {management }},
$$

where $R$ is the transponder operation baud rate, $n$ is the active laser(s) number(s), $P_{\text {static }}$ is the total number of elements with fixed (static) power consumption, and $P_{\text {dynamic }}$ is the parameter that captures dynamic power consumption. The aggregate power consumed by the BVT transponder is increased by $P_{\text {management }}$, which is fixed at $20 \%$ of transponder total power consumption ${ }^{[17]}$. In addition, we fix the values of $P_{\text {static }}$ and $P_{\text {dynamic }}$ to 148 and 5.6, respectively, which are evaluated so as to fit linearly to 100 and 400 Gbit/s. Tab. 2 shows the power consumption(s) of the BVT model with various transmission options. The TR values shown in Tab. 2 have been calculated for $n$ bits, where $n=4,8$, or 16 for DPQPSK, DP-8-QAM, and DP-16-QAM formats, respectively.

Table 2 Power consumption values of various BVTs

\begin{tabular}{ccccc}
\hline capacity & $\begin{array}{c}\text { transmission } \\
\text { reach }(\mathrm{km})\end{array}$ & data slots & $\begin{array}{c}\text { guardband } \\
\text { slots }\end{array}$ & $\begin{array}{c}\text { power } \\
(\mathrm{W})\end{array}$ \\
\hline \multirow{4}{*}{$40 \mathrm{Gbit} / \mathrm{s}$} & 3900 & 4 & 1 & 833.5 \\
& 2800 & 3 & 1 & 833.5 \\
& 2300 & 2 & 1 & 833.5 \\
& 1700 & 1 & 1 & 833.5 \\
\hline \multirow{4}{*}{$100 \mathrm{Gbit} / \mathrm{s}$} & 4200 & 4 & 1 & 1240 \\
& 2800 & 3 & 1 & 1240 \\
& 1700 & 2 & 1 & 1240 \\
& 1600 & 1 & 1 & 1240 \\
& 620 & 8 & 1 & 1730 \\
& 490 & 6 & 1 & 1730 \\
& 410 & 4 & 1 & 1600 \\
\hline
\end{tabular}




\section{Algorithm details}

During the network planning procedure, we use the network topology and set of traffic demands as inputs. Specifically, we are given (i) a network topology $G(V, E)$ that comprises a set of $V$ nodes and $E$ links, (ii) an a-priori traffic matrix $T=\left[\Lambda_{s-d}\right]$ that consists of traffic having total requests in Gbit/s of $\Lambda_{s-d}$ between a source-destination pair, and (iii) physical lengths $D_{l}$ of the links $l \in E$. The aim is the servicing of all traffic requests and lowering of the cost and power consumption in both fixedand flexi-grid networks. This corresponds to choosing the right transponder type for each connection in the MLR network and assuming the utilization of an individual type of tunable transponder configuration for every connection. The network planning issues are known to be NP-hard ${ }^{[2,13]}$; hence, searching for absolute optimums for realistic topologies with multiple nodes and/or links and high traffic loads is time consuming. Therefore, we resort to the use of heuristic algorithms that (i) serve the requests in the static traffic matrix individually, as per a fixed order; (ii) store the selections made for requests that were served previously so as to prevent wavelength contention; and (iii) additively evaluate the cost/power consumption of the entire network. Moreover, the power and cost consumption are highly dependent on the order of demand serving, since choices made for one connection may differ at a later instant if the route that is chosen is congested, thus requiring the addition of add/drop terminals whereas the avoided route is relatively empty ${ }^{[13]}$. As ordering is of importance, to search among various orderings, we use SA (Simulating Annealing) metaheuristic. The algorithms used are as follows:

1. For the MLR case, we use the heuristic variation of our previous MLR algorithm ${ }^{[38]}$, called the PA-RWA (Power-Aware RWA) algorithm. PA-RWA pre-calculates the $k$-SPs ( $k$-Shortest Paths) for each demand, and for every route, given different types of transponders with their respective TRs, at the previous link node, it allocates a regenerator which increases path longevity compared to the given TR.
Before moving to serve the next demand, PA-RWA accounts for node architecture, NIs utilized up to the current point and points of regeneration of every $k$ paths, and selects the route resulting in the lowest increase of network power consumption.

2. For the SLR case, PA-RWA is downgraded by changing the bit-rate value $r_{k}$ to a pre-defined bit rate value $r$. Hence, the same calculations are made as in the MLR case; however, for a specific SLR transponder with a given TR, the path that minimizes the additive power consumption of serving the current demand is selected before moving to the next demand.

3. For the flexi-grid case, we use the RMLSA simulated-annealing algorithm detailed in Ref. [13]. We extended the heuristic algorithm for power awareness and call it the PA-RMLSA (Power-Aware RMLSA) algorithm. As input, PA-RMLSA is supplied with viable transmission configurations of different transponders in the form of tuples $t=$ $\left\{l_{t}, r_{t}, b_{t}, g_{t}, c_{t}, p_{t}\right\}$, as shown in Tab. 1. PA-RMLSA includes the power consumption of serving a demand in its weighted multiobjective cost. It calculates $k$ SPs for every request, and subsequently, for these individual routes and the viable tuple for transmission, it evaluates the points of regeneration. The execution time is slowed owing to the many solutions that exist for each demand; hence, as an additional phase, over the same path, compared to other tuples, PA-RMLSA prunes those tuples that consume more power and spectrum ${ }^{[40]}$. Then, it calculates the additive network power consumed by each option, accounting for the requests that have been served up to that point, and chooses the pair of tuple-path that minimizes a weighted combination of utilized additive spectrum and power.

\section{Simulation results and discussion}

Within the minimization function of our simulations, we neglect the spectrum used and set the weighting coefficient for power consumption to minimize the power only. However, as an extension, we also show the results when the spectrum is minimized exclu- 


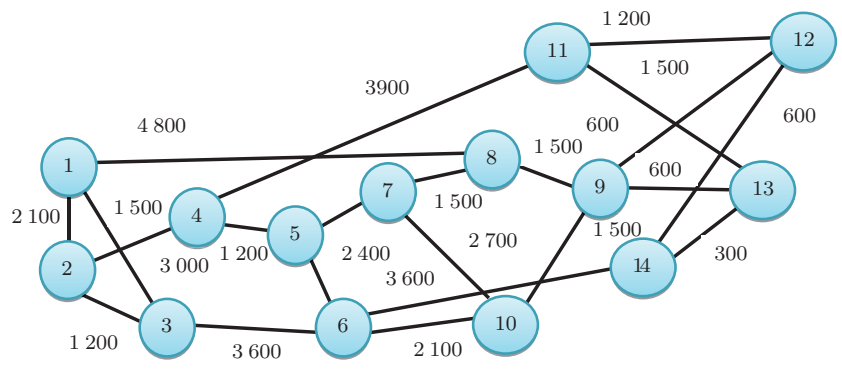

Figure 4 NSFNet topology

sively (see section 5.3). We consider two realistic backbone network topologies: (i) the 14-node and 21 (bidirectional)-link NSFNet ${ }^{[1]}$ (shown in Fig. 4), with a mean nodal degree and average link length of 3.0 and $1086 \mathrm{~km}$, respectively, and (ii) the 11-node and 26 (bidirectional)-link COST239 ${ }^{[37]}$ (shown in Fig. 5), with a mean nodal degree and average link length of 3.2 and $439 \mathrm{~km}$, respectively. For the simulations, we consider the corresponding traffic matrices of both topologies, as given in Refs. [1,39]. For the COST239 topology, the traffic matrix amounts to a total of $1 \mathrm{Tbit} / \mathrm{s}$ of traffic requests, whereas for the NSFNet topology, we multiply the base traffic matrix by a constant (set to "3" in our study) to obtain a total of 3 Tbit/s of traffic requests. Further, in the simulations, the required resources are calculated so as to completely accommodate the requested demand. For the fixed-grid network case, wavelengths are assumed to be supported, and TR values are similar to those for the cases of the SLR and MLR networks, whereas for the case of the flexi-grid network with a spectrum slot of $12.5 \mathrm{GHz}$, spectrum slots are available.

The performance of the simulated-annealing algorithm depends on the following parameters, which can also be tuned: (i) cooling rate, (ii) starting temperature coefficient, and (iii) number of iterations. Initially, we conducted we conducted tuning of the RMLSA simulated-annealing algorithm, which consisted of the evaluation of different cases with various values of demands, for cooling rate and starting temperature coefficient between 0.9 0.999 9 and 0.05 1, respectively. In regard to our optimization objective,

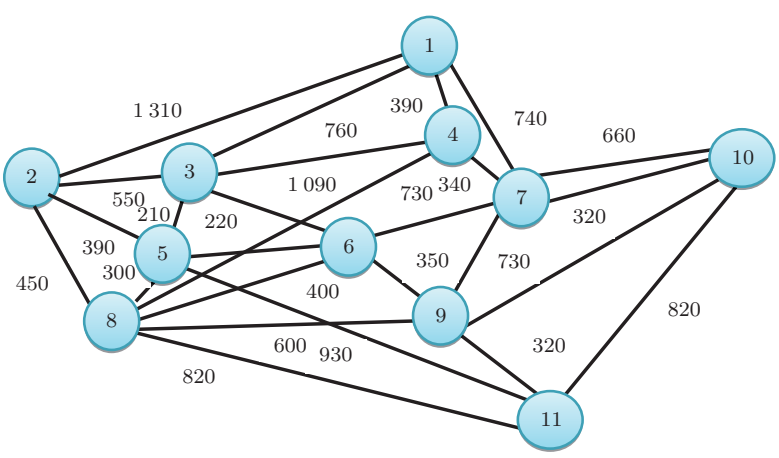

Figure 5 COST239 topology

the values offering the best performance are shown in Tab. 3. The values shown in Tab. 3 are used to obtain further results in the subsequent subsections, and the numbers of iterations chosen show satisfactory solution convergence and reasonable run times (up to $3400 \mathrm{~s}$ ).

Table 3 The RMLSA simulated-annealing algorithm tuning procedure results

\begin{tabular}{cccc}
\hline networks & cooling rate & $\begin{array}{c}\text { starting } \\
\text { temperature } \\
\text { coefficient }\end{array}$ & $\begin{array}{c}\text { numbers of } \\
\text { iterations }\end{array}$ \\
\hline COST239 & 0.99 & 0.25 & 6000 \\
NSFNet & 0.99 & 0.05 & 5000 \\
\hline
\end{tabular}

\subsection{Cost-efficiency}

Fig. 6 shows the variation of CapEx with average TL (Traffic Load) (demand) for the COST239 and NSFNet topologies. The following points should be noted in regard to the results presented in this subsection: (i) the algorithms used optimized CapEx indirectly as they were formulated for optimization of consumed power, (ii) for the CapEx calculations the costs of the BVTs and WSSs used in the flexigrid case are assumed to be $35 \%$ more than those of the corresponding fixed-grid network equipment ${ }^{[21]}$.

It can be seen from Fig. 6 that for both topologies, compared to the MLR and flexi-grid networks the SLR networks always consume more CapEx for any given TL value. Comparing the MLR and flexi-grid networks, it is seen that (i) for the COST239 topology, the flexi-grid network consumes more CapEx than the MLR network for all TL values, and (ii) 


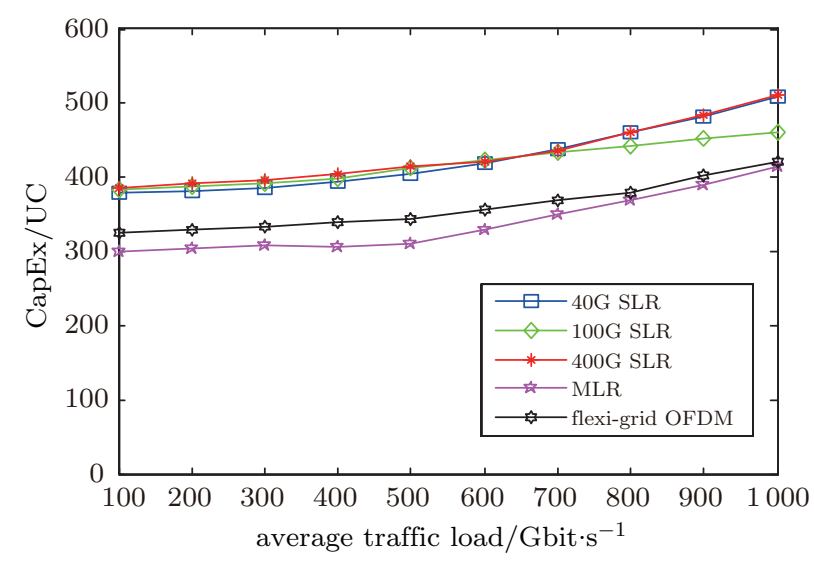

(a)

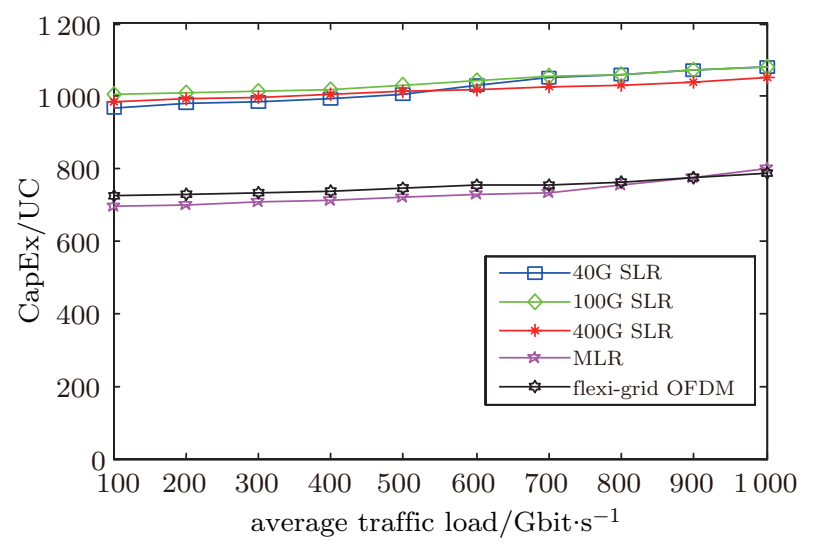

(b)

Figure 6 The variation of CapEx with average traffic load: (a) COST239 topology; (b) NSFNet topology

for the NSFNet topology, for higher values of TL, the flexi-grid network consumes less CapEx than the MLR network. This occurs due to the larger variations in length of routes and requests for the NSFNet topology, for which various transponder configurations can be utilized at high TLs. It can be concluded that owing to the greater diversity of the network and its corresponding traffic, the use of the flexi-grid network provides more benefits than use of the fixed-grid network.

\subsection{Power-efficiency}

Fig. 7 shows the power consumed by both topologies as a function of average TL. It is seen from the figures that for both topologies (i) at heavy TLs, the flexi-grid network consumes the least power; (ii) at medium TLs, the MLR and flexi-grid networks consume approximately the same amount of power, and (iii) at low TLs the MLR network consumes slightly less power than the flexi-grid network. The aforementioned occurs because at higher TLs the flexigrid network is able to exploit a greater number of transmission options. In addition, for both topologies, the power consumed by the SLR cases is always higher than the MLR and flexi-grid cases.

For the COST239 topology, comparing all the SLR cases, it is observed that for low TLs, the $40 \mathrm{Gbit} / \mathrm{s}$ SLR network consumes less power than the 100 and 400 Gbit/s SLR networks. However, as the TL in- creases, the $100 \mathrm{Gbit} / \mathrm{s}$ SLR network begins to consume less power than the $40 \mathrm{Gbit} / \mathrm{s}$ SLR network, and at very high TLs, the $400 \mathrm{Gbit} / \mathrm{s}$ SLR network power consumption begins to merge with that of the 100 Gbit/s SLR network. Hence, it can be predicted that at even higher TL values, the $400 \mathrm{Gbit} / \mathrm{s}$ SLR network would consume less power than both the 40 and $100 \mathrm{Gbit} / \mathrm{s}$ SLR networks. On the other hand, for the NSFNet topology, the power consumed by the SLR networks is much higher than that consumed by the MLR and flexi-grid cases in the same network. This occurs owing to larger variations in length of routes and requests for the NSFNet topology, which makes it ineffective for serving traffic with an individual type of transponder or regenerator, as exercised for the SLR networks. Hence, it can be concluded from Fig. 7 that the flexi-grid and MLR networks exhibit similar power consumptions for almost all TLs and outperform all SLR network options, owing to the capability of exploiting different transmission options to fine-tune transmission rates to the actual capacity requested. For SLR networks, the network characteristics and the capacity requested between each node pair considerably affect the power consumption.

Following our previous study ${ }^{[17]}$, we use power efficiency (specified in units of $\mathrm{W} / \mathrm{Gbit}^{-1} \mathrm{~s}^{-1}$ ) as another metric to clearly depict the manner in which the physical topology and power consumption for the requested capacity service are associated. Fig. 8 


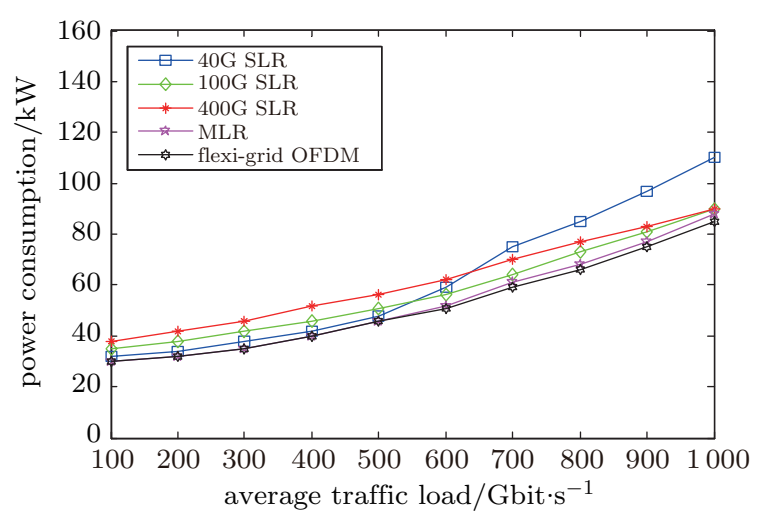

(a)

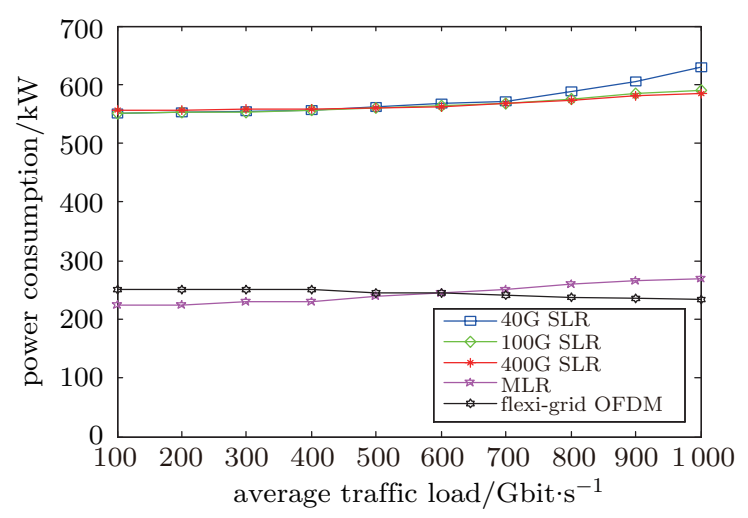

(b)

Figure 7 Total power consumption versus average traffic demand: (a) COST239 topology; (b) NSFNet topology

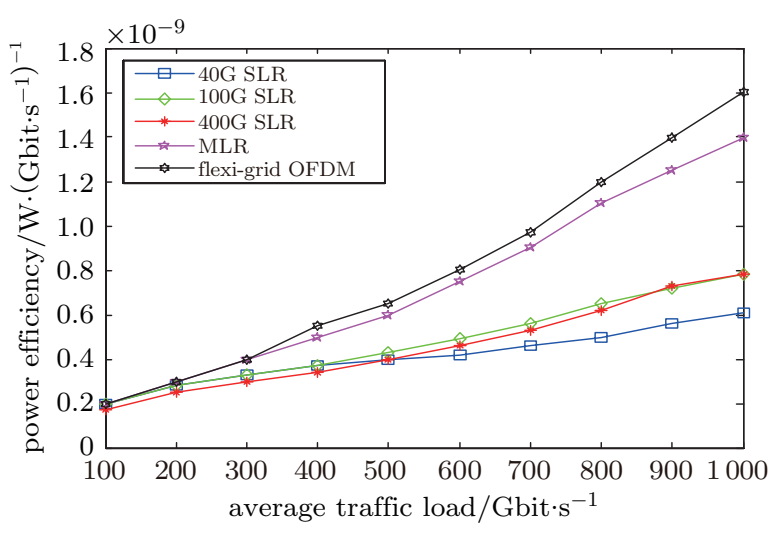

(a)

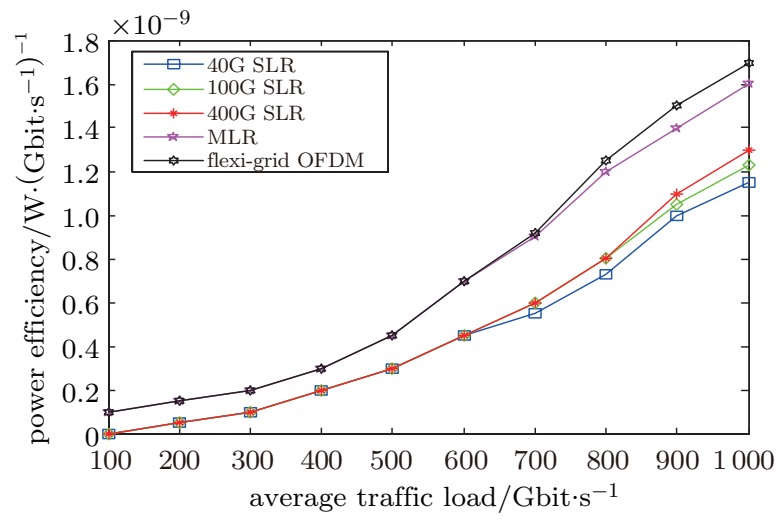

(b)

Figure 8 Power efficiency versus average traffic demand: (a) COST239 topology; (b) NSFNet topology

shows the variation of power efficiency with average TL. For the COST239 topology, it can be observed that compared to the MLR networks, there is a slight difference in power efficiency between the flexi-grid and MLR networks owing to the finer granularities of the various modulation formats. Among the SLR networks, it is observed that the $100 \mathrm{Gbit} / \mathrm{s}$ SLR shows superior power efficiency for almost all the TLs. However, the power efficiency in the 100 and $400 \mathrm{Gbit} / \mathrm{s}$ SLR networks remains inferior to both the MLR and flexi-grid networks. For such networks, the longer distances traversed by few routes leads to the placement of a large number of regenerators, which, in turn, results in increased power consumption for both the 400 and $100 \mathrm{Gbit} / \mathrm{s}$ networks owing to their TRs being 790 and $2100 \mathrm{~km}$, respectively. The difference for the NSFNet topology occurs in the MLR network which closely traces the flexi-grid network for almost all the TLs. In addition, in the NSFNet topology, many long-distance paths exist, leading to the placement of regenerators that increase the consumed power. With the elasticity of the flexi-grid network, long distances of few network routes are adjusted owing to the various formats and rates of subcarrier modulation, offering maximum TR. For these paths, the MLR network is able to utilize only the $40 \mathrm{Gbit} / \mathrm{s}$ wavelength, offering $2300 \mathrm{~km}$ TR.

For both topologies, we also present results detailing the share of each component in the aggregate power consumption of the flexi-grid and MLR networks. Fig. 9(a) shows that in the WDM (Wavelength Division Multiplexed) layer, for the flexi-grid COST239 topology, at a low TL value of $100 \mathrm{Gbit} / \mathrm{s}$, approximately $75 \%$ of the aggregate power is consumed by the transponders. However, as the TL in- 


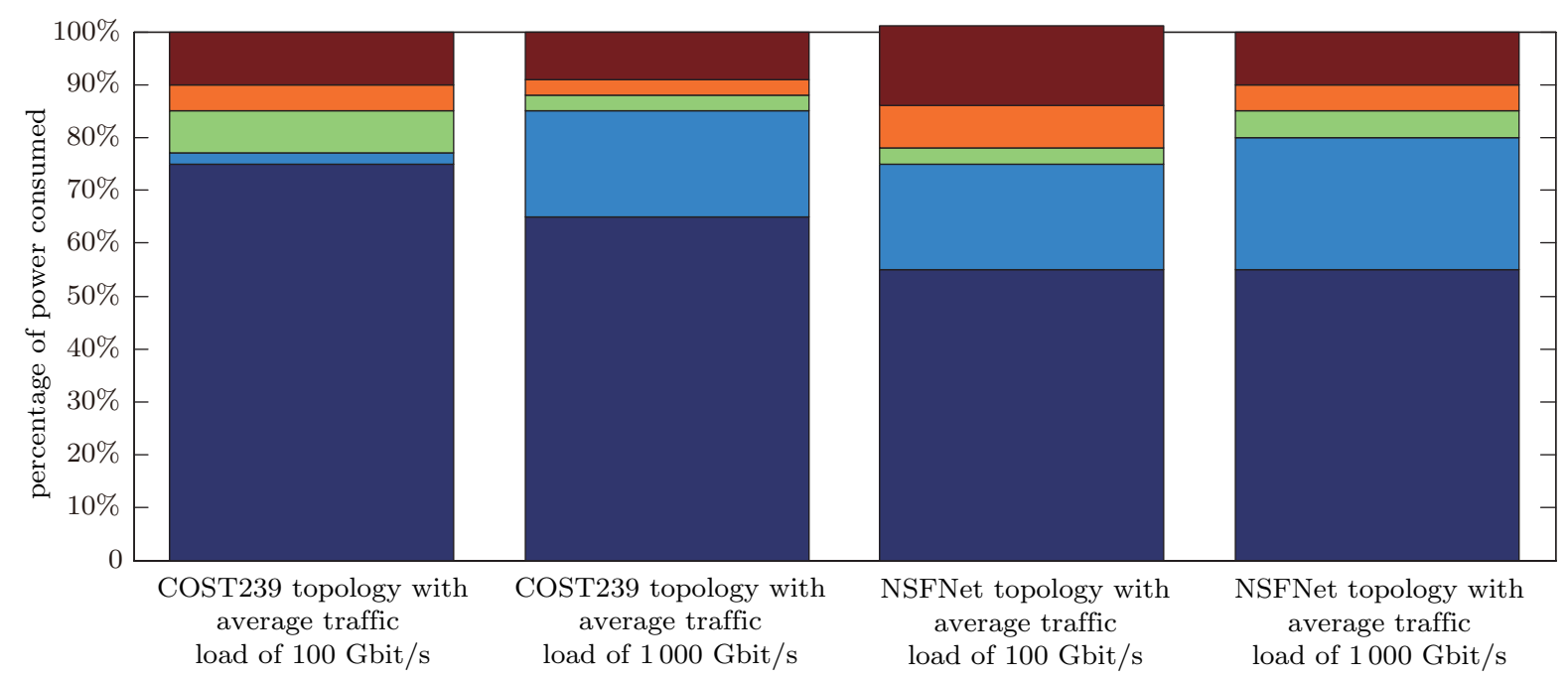

(a)

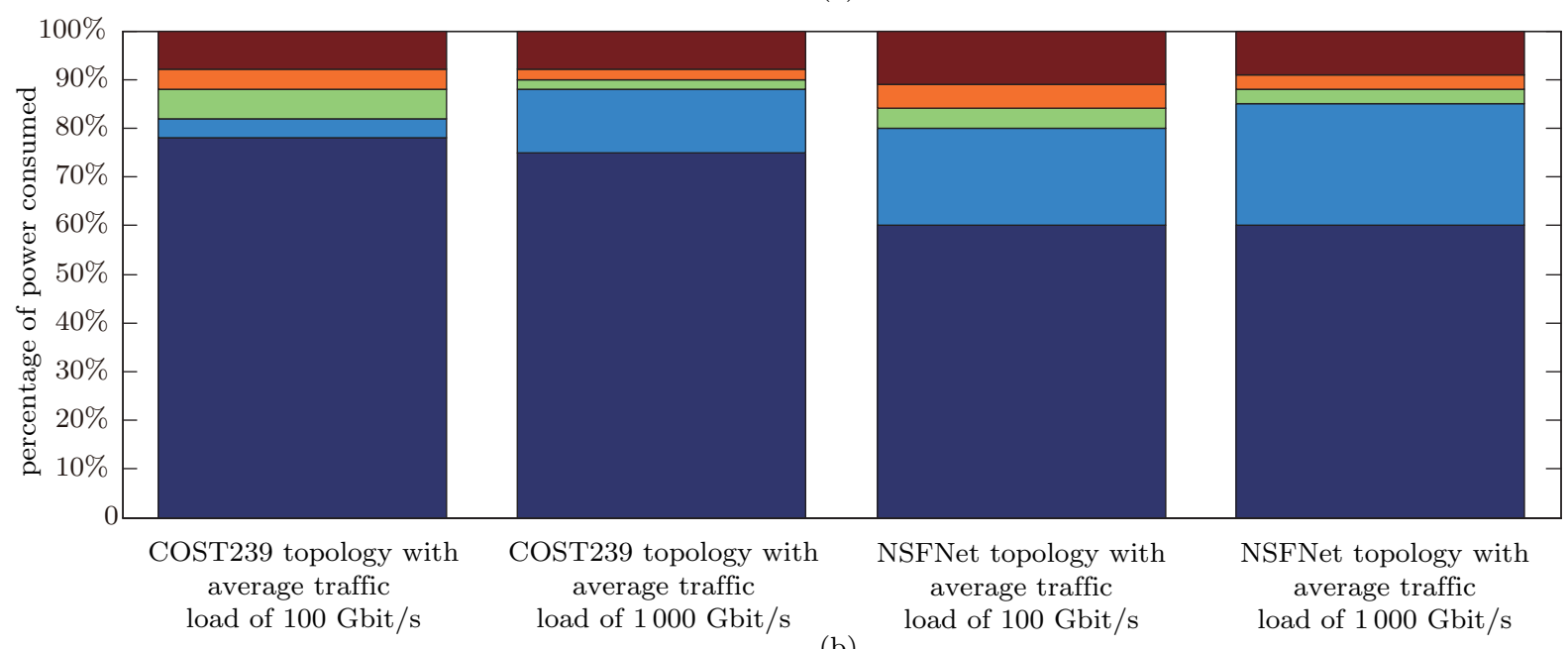

(b)
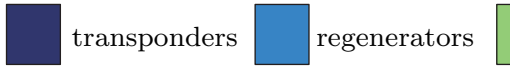

terminal

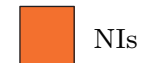

EDFAs

Figure 9 Consumed power for every component: (a) flexi-grid network; (b) MLR network

creases to $1000 \mathrm{Gbit} / \mathrm{s}$, a larger number of regenerators are required, which changes the network power profile since the regenerators consume an increasing portion of the power. In contrast, for the NSFNet topology, which consists of multiple routes with long distances and a traffic matrix with multiple low bitrate requests, many regenerators are used even at lower TL value of $100 \mathrm{Gbit} / \mathrm{s}$. Hence, less power (approximately 60\%) is consumed by the transponders; however, the regenerators are observed to consume more power compared to the COST239 topology case. Considering the MLR network, for both topologies similar results are obtained as for the flexi- grid network, as shown in Fig. 9(b).

Fig. 10 shows the maximum spectrum utilization as a function of average traffic demand for the COST239 and NSFNet topologies. It can be seen from the figure that for both the topologies, the flexigrid network uses the least amount of spectrum since it provides finer granularity and multiple options for transmission. The MLR network also provides multiple modes of transmission but consumes more spectrum than the flexi-grid network owing to the fewer transmission options available. Comparing the SLR cases, it is seen that that for the COST239 topology, the $400 \mathrm{Gbit} / \mathrm{s}$ SLR network consumes the least 


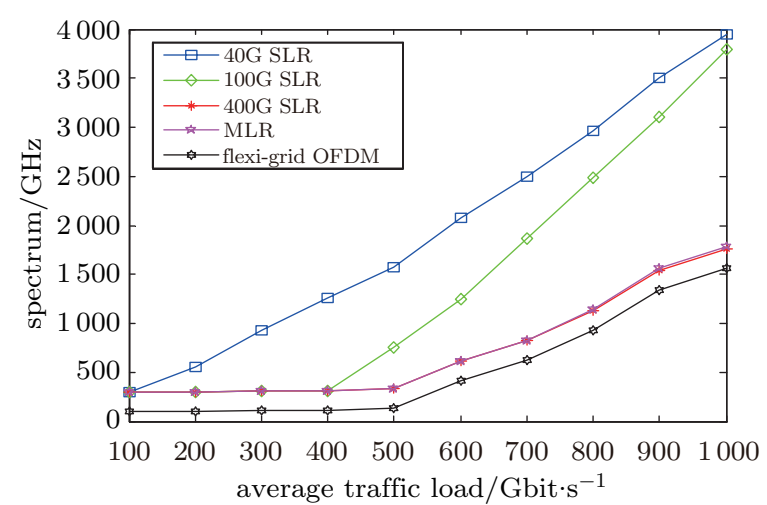

(a)

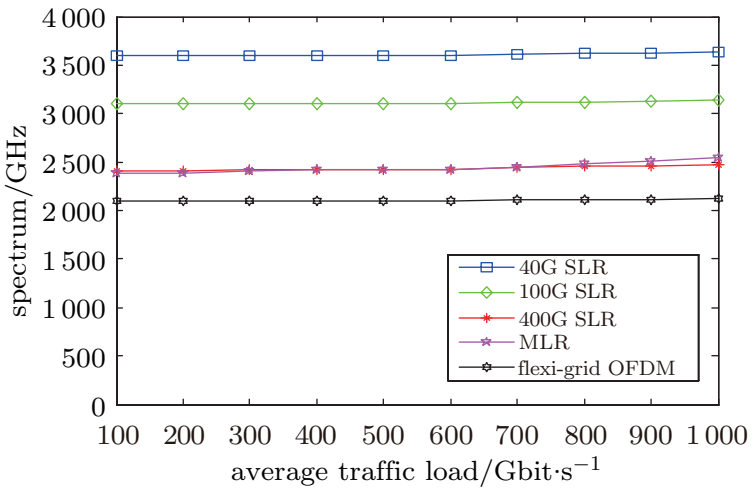

(b)

Figure 10 Maximum spectrum used versus average traffic demand: (a) COST239 topology; (b) NSFNet topology

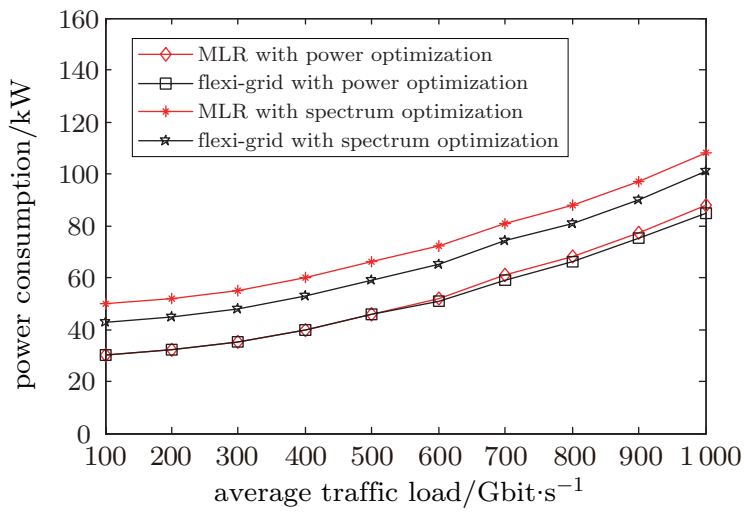

(a)

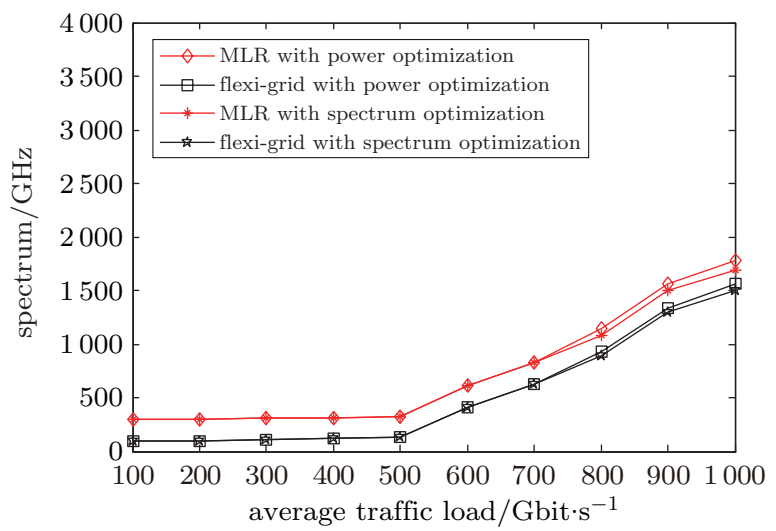

(b)

Figure 11 For the COST239 topology: (a) power savings versus variation of average traffic load; (b) spectrum occupancy versus variation of average traffic load

amount of spectrum since the topology link lengths are small, enabling the support of high bit-rate transparent transmissions. This in turn requires many regenerators that relax the wavelength continuity constraint, hence achieving better utilization of the spectrum. However, the use of many regenerators increases the power and CapEx consumption, which explains the higher power and CapEx consumption of the $400 \mathrm{Gbit} / \mathrm{s}$ SLR network for the COST239 topology.

As an extension, for the flexi-grid network, apart from minimizing the power as an objective we also evaluated the objective of minimizing the use of spectrum (see Figs. 11 and 12). From Fig. 11 it can be observed that for the COST239 topology, compared to where the objective is minimization of the spectrum, notable saving in power is achieved when the objec- tive is set to minimization of power. Moreover, to achieve the aforementioned, only a modest forfeiture of the spectrum is incurred, as the use of spectrum is approximately the same as the best utilization that can be achieved when the objective of minimizing the spectrum is used. For the NSFNet topology, it can be seen that owing to its increased variability of links and traffic requests, to obtain substantial savings in power, considerable forfeiture of spectrum is required. Thus, there exists a power and spectrum minimization trade-off that is dependent on the network characteristics. Finally, the following two major conclusions can also be drawn from Figs. 11 and 12:

1. For cases where the objective is network power minimization, a larger amount of spectrum is used owing to (i) specific power savings (e.g., in number 


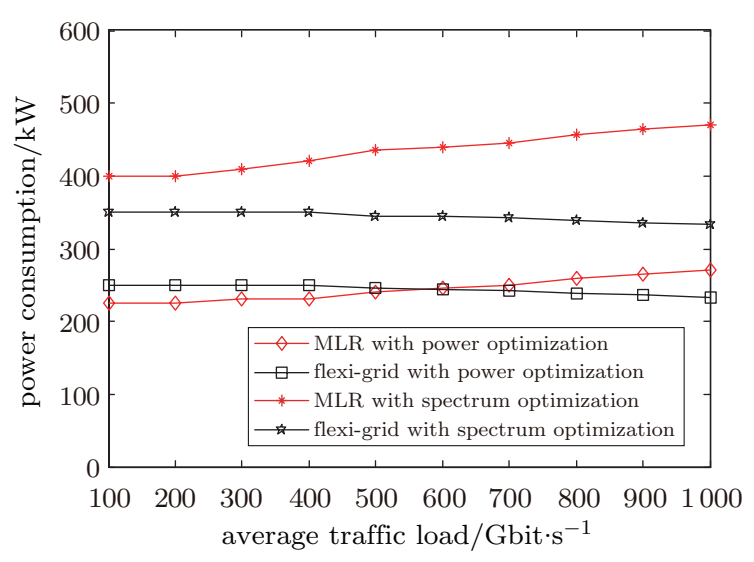

(a)

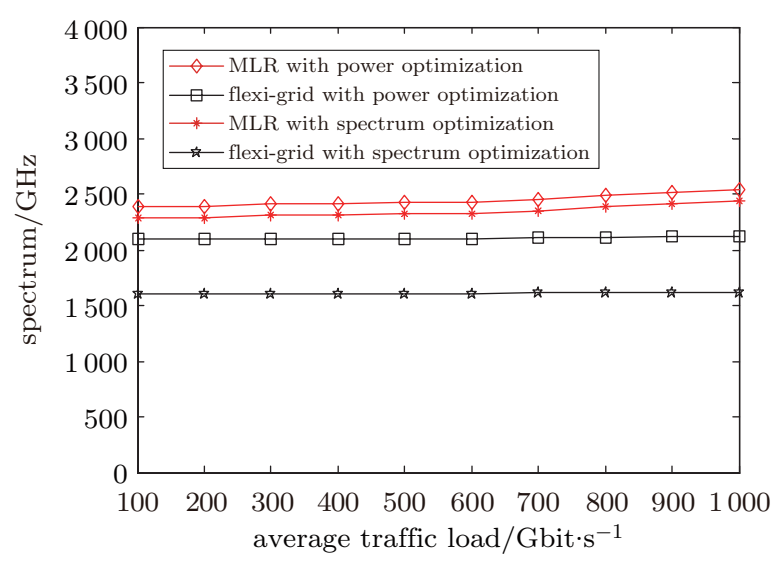

(b)

Figure 12 For the NSFNet topology: (a) power savings versus variation of average traffic load; (b) spectrum occupancy versus variation of average traffic load

of add/drop terminals), which are obtained without the use of similar spectrum slots, or (ii) various BVT configurations that trade off power with consumed spectrum.

2. For cases where the objective is network spectrum minimization, a higher amount of network power is consumed, which makes such an objective function power-unaware.

\section{Conclusion and future work}

In this work, for fixed- and flexi-grid networks, we developed models to identify analytically the major factors related to cost and power consumption in such networks. The models were used to compare techniques for improving the $\mathrm{CE}$ and $\mathrm{PE}$ in fixedand flexi-grid networks. We conducted a $\mathrm{CE}$ and $\mathrm{PE}$ comparative analysis for OFDM- and MLR/SLRbased networks, and also evaluated the cost and power consumed by different components in the optical layer, considering realistic networks. The results show that flexi-grid networks outperform fixedgrid networks in terms of cost and power, and the extra equipment cost incurred due to less efficient use of spectrum can be reduced by switching to a flexi-grid network. The results also indicate that (i) lower power consumption per bit is obtained when the networking solution ensures finer bit-rate granularity, and (ii) there exists a power and spectrum minimization trade-off that is dependent on network characteristics. In our future research, we aim to utilize actual electricity prices that play a key role in OpEx minimization, a factor which has not so far been considered in network planning algorithms. We aim to determine whether accounting for actual location-based electricity prices, which vary in the continental networks, results in any major monetary savings.

\section{References}

[1] A. Nag, M. Tornatore, B. Mukherjee. Optical network design with mixed line rates and multiple modulation formats [J]. IEEE/OSA journal of lightwave technology, 2010, 28(4): 466-475.

[2] S. P. Singh, S. Sengar, R. Bajpai, et al. Next-generation variable-line-rate optical WDM networks: Issues and challenges [J]. Journal of optical communication, 2013, 34(4): 331-350.

[3] M. Jinno, B. Kozicki, H. Takara, et al. Distanceadaptive spectrum resource allocation in spectrumsliced elastic optical path network [J]. IEEE communications magazine, 2009, 48(8): 138-145.

[4] M. Jinno, H. Takara, B. Kozicki, et al. Spectrumefficient and scalable elastic optical path network: Architecture, benefits, and enabling technologies [J]. IEEE communications magazine, 2009, 47(11): 66-73.

[5] O. Gerstel, M. Jinno, A. Lord, et al. Elastic optical networking: A new dawn for the optical layer [J]. IEEE communications magazine, 2012, 50(2): S12-S20.

[6] A. Morea, A. F. Chang, O. Rival. Impact of transparent network constraints on capacity gain of elastic channel spacing [C]//IEEE Optical Fiber Communication/National Fiber Optic Engineers Conference 
(OFC/NFOEC), 2011: JWA62.

[7] O. Rival, A. Morea. Cost-efficiency of mixed 10$40-100 \mathrm{~Gb} / \mathrm{s}$ networks and elastic optical networks [C]//IEEE Optical Fiber Communication/National Fiber Optic Engineers Conference (OFC/NFOEC), 2011: OTuI4.

[8] X. Dong, T. E. H. ElGorashi, J. M. H. Elmirghani. Energy efficiency of optical OFDM-based networks [C]//Proceedings of IEEE ICC, 2013: 4131-4136.

[9] J. Lopez, Y. Ye, I. T. Monroy. Energy efficiency in flexible bandwidth optical networks [C]//International Conference on the Network of the Future (NOF), 2011: 107-111.

[10] J. Lopez, Y. Ye, V. Lopez, et al. On the energy efficiency of survivable optical transport networks with flexiblegrid [C]//IEEE European Conference and Exhibition on Optical Communication (ECOC), 2012: P5.05.

[11] H. Khodakarami, B. P. Gopalakrishna, B. Sedighi, et al. Flexible optical networks: An energy efficiency perspective [J]. IEEE/OSA journal of lightwave technology, 2014, 32(21): 3356-3367.

[12] ITU-T Recommendation G.694.1. Spectral grids for WDM applications: DWDM frequency grid [S]. 2012.

[13] K. Christodoulopoulos, I. Tomkos, E. A. Varvarigos. Elastic bandwidth allocation in flexible OFDM-based optical networks $[\mathrm{J}]$. IEEE/OSA journal of lightwave technology, 2011, 29(9): 1354-1366.

[14] SMART 2020: Enabling the low carbon economy in the information age, GESI [R]. 2008.

[15] Cisco visual networking index: Forecast and methodology 2012-2017. Cisco White Paper [R]. 2013.

[16] F. Idzikowski, S. Orlowski, C. Raack, et al. Saving energy in IP-over-WDM networks by switching off line cards in low-demand scenarios [C]//IEEE Optical Network Design and Modeling (ONDM), 2010: 1-3.

[17] S. Iyer, S. P. Singh. Spectral and power-efficiency investigation in single and multi-line-rate optical wavelength division multiplexed (WDM) networks [J]. Photonic network communications, 2017, 33(1): 39-51.

[18] B. Collings. New devices enabling software-defined optical networks $[\mathrm{J}]$. IEEE communications magazine, 2013, 51(3): 66-71.

[19] Y. Li, L. Gao, G. Shen, et al. Impact of ROADM colorless, directionless, and contentionless (CDC) features on optical network performance [J]. IEEE/OSA journal of optical communications and networking, 2012, 4(11): B58-B67.

[20] F. Rambach, B. Konard, L. Dembeck, et al. A multilayer cost model for metro/core networks [J]. IEEE/OSA journal of optical communications and networking, 2013, 5(3): 210-225.

[21] FP 7-STRONGEST project, D2.1. Efficient and optimized network architecture: Requirements and reference scenarios [EB/R]. http://www.ict-strongest.eu.

[22] Finisar Corporation. ROADMs and wavelength man- agement [EB/OL]. https://www.finisar.com/roadmswavelength-management/10wsaaxxfll.

[23] Finisar Corporation. Optical transceivers [EB/OL]. https://www.finisar.com/optical-transceivers/ftlc3321$\mathrm{x} 3 \mathrm{nl}$.

[24] ADVA Packet Optical Convergence, Juniper. White paper [EB/R]. https://www.juniper.net/assets/kr/kr/ local/pdf/whitepapers/2000582-en.pdf.

[25] W. V. Heddeghem, F. Izdikowski, W. Vereecken, et al. Equipment power consumption in optical multilayer networks $[\mathrm{EB} / \mathrm{R}]$. Source Data. Berlin (TKN). Report Number: IBCN-12-001-01, http://www.tkn.tuberlin.de/fileadmin/fg112/Internals/idzi12Equipmentpowercons.pdf, 2012: 1-28.

[26] ADVA. FSP 3000 coherent transponders. Fact sheet [R]. 2012.

[27] Ciena: F10-T 10G transponder. Datasheet [EB/R]. http://www.ciena.com/products/f10-t/tab/features/, 2011.

[28] Lementum. Wavelength selective switches [EB/OL]. https://www.lumentum.com/en/products/trueflex-1x9 -wavelength-selective-switch.

[29] Lementum. Wavelength selective switches [EB/OL]. https://www.lumentum.com/en/products/trueflex-twin-high-port-countwavelength-selective-switch-twin-wss.

[30] I. Dedic. High-speed CMOS DSP and data converters $[\mathrm{C}] / /$ IEEE Optical Fiber Communication/National Fiber Optic Engineers Conference (OFC/NFOEC), Workshop, Transmission Subsystems and Network Elements, 2011: OTuN1.

[31] I. Dedic. 56Gs/s ADC enabling 100GbE [C]//IEEE Optical Fiber Communication/National Fiber Optic Engineers Conference (OFC/NFOEC), 2010: 1-3.

[32] R. Bouziane, P. Milderet, R. Koutsoyannis, et al. Design studies for an ASIC implementation of an optical OFDM transceiver [C]// IEEE European Conference and Exhibition on Optical Communication (ECOC), 2010: 1-3.

[33] F. Idzikowski. Power consumption of network elements in IP over WDM networks [EB/R]. TU Berlin, Germany, Technical Report, 2010.

[34] Transmode TM-series data sheet [EB/R]. http://www. transmode.com/, 2010.

[35] Fujistu 2015 [EB/OL]. http://www.fujitsu.com/jp/ group/foc/en/resources/news/press-releases/2015/20150319-3.html.

[36] A. Napoli, M. Bohn, D. Rafique, et al. Next generation elastic optical networks: The vision of the european research project IDEALIST [J]. IEEE communications magazine, 2015, 53(2): 152-162.

[37] IDEALIST Project. Elastic optical network architecture: Reference scenario, cost and planning [R]. Deliverable D1.1, 2013.

[38] S. Iyer, S. P. Singh. Investigation of launch power and regenerator placement effect on the design of mixed-linerate (MLR) optical WDM networks [J]. Photonic net- 
work communications, 2017.

[39] A. Nag, T. Wang, B. Mukherjee. Robust design of spectrum-efficient green optical backbone networks [J]. IEEE/OSA journal of lightwave technology, 2013, 31(7): 1138-1144.

[40] K. Christodoulpoulos, P. Soumplis, E. A. Varvarigos. Planning flexible optical networks under physical layer constraints [J]. IEEE/OSA journal of optical communications and networking, 2013, 5(11): 1296-1312.

\section{About the authors}

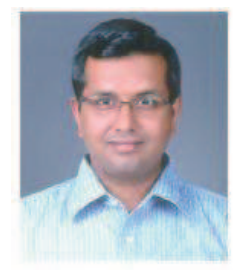

Sridhar Iyer [corresponding author] received the B.E. degree in electronics and telecommunications engineering from Mumbai University, India in 2005, M.S degree in electrical and communication engineering from New Mexico State University, U.S.A in 2008, and the Ph.D. degree from Delhi University, India in 2017. He worked as an assistant professor in the Department of ECE at NIIT University, and Christ University, India between 2012-2016. Currently he is an associate professor in the Department of ECE, Jain College of Engineering, India. His research inter- ests include the architectural, algorithmic, and performance aspects of the optical networks, with current emphasis on efficient design and resource optimization in the flexi-grid elastic optical networks. Dr. Iyer has published over 30 peerreviewed articles in the aforementioned areas. (Email: sridhariyer1983@gmail.com)

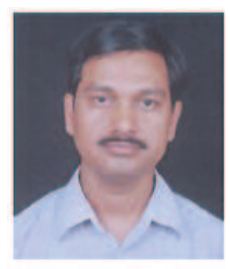

Shree Prakash Singh received received his B.E. degree in electronics and communication engineering from MMM Engineering College, Gorakhpur, India, in 1990 and M.Tech. degree from REC, Kurukshetra, India, in 1994. He holds a doctoral degree in optical networks from the Indian Institute of Technology, Delhi (IITD), India, in 2007. During 1994-2000, he worked as lecturer in the Department of Electronics and Computer Engineering at REC Kurukshetra. In October 2000, he joined as lecturer in the Department of Electronics, Netaji Subhas Institute of Technology (formally known as Delhi Institute of Technology New Delhi). Presently he is a professor at the Division of Electronics and Communication Engineering, Netaji Subhas Institute of Technology, New Delhi. His current area of research is optical networks, optical wireless communication. (Email:sps_nsit@yahoo.co.uk) 